Research Paper

\title{
Developing a multivariable normal tissue complication probability model to predict late rectal bleeding following intensity-modulated radiation therapy
}

\author{
Chun-Chieh Huang1,2, Pei-Ju Chao,1,3, Shih-Sian Guo1,3, Chong-Jong Wang1, Hao-Lun Luo ${ }^{4}, Y^{1}$-Li Su ${ }^{5}$, \\ Tsair-Fwu Lee ${ }^{1,3,6 凶}$, Fu-Min Fang ${ }^{1 凶}$ \\ 1. Department of Radiation Oncology, Kaohsiung Chang Gung Memorial Hospital and Chang Gung University College of Medicine, Kaohsiung, Taiwan \\ 2. Graduate Institute of Clinical Medical Sciences, College of Medicine, Chang Gung University, Taoyuan, Taiwan \\ 3. Medical Physics and Informatics Laboratory of Electronics Engineering, National Kaohsiung University of Science and Technology, Kaohsiung, Taiwan \\ 4. Department of Urology, Kaohsiung Chang Gung Memorial Hospital and Chang Gung University College of Medicine, Kaohsiung, Taiwan \\ 5. Division of Hematology-Oncology, Department of Internal Medicine, Kaohsiung Chang Gung Memorial Hospital and Chang Gung University College of \\ Medicine, Kaohsiung, Taiwan \\ 6. Graduate Institute of Clinical Medicine, Kaohsiung Medical University, Kaohsiung, Taiwan
}

$\triangle$ Corresponding authors: Fu-Min Fang, MD, PhD., Department of Radiation Oncology, Kaohsiung Chang Gung Memorial Hospital, No.123, Dapi Rd., Niaosong Dist., Kaohsiung 833, Taiwan. Tel.: +886-9-75056452; Fax: +886-7-7322813; Email: fang2569@cgmh.org.tw. Tsair-Fwu Lee, PhD., Medical Physics and Informatics Laboratory of Electronics Engineering, National Kaohsiung University of Science and Technology, No.415, Jiangong Rd., Sanmin Dist., Kaohsiung 807, Taiwan. Tel.: +886-7-3814526 ext. 5657; Fax: +886-7-3811182; Email: tflee@kuas.edu.tw

(c) Ivyspring International Publisher. This is an open access article distributed under the terms of the Creative Commons Attribution (CC BY-NC) license (https://creativecommons.org/licenses/by-nc/4.0/). See http://ivyspring.com/terms for full terms and conditions.

Received: 2018.08.30; Accepted: 2019.04.27; Published: 2019.06.02

\begin{abstract}
Purpose: To develop a multivariable normal tissue complication probability (NTCP) model to predict moderate to severe late rectal bleeding following intensity-modulated radiation therapy (IMRT).

Methods and materials: Sixty-eight patients with localized prostate cancer treated by IMRT from 2008 to 2011 were enrolled. The median follow-up time was 56 months. According to the criteria of D'Amico risk classifications, there were 9, 20 and 39 patients in low, intermediate and high-risk groups, respectively. Forty-two patients were combined with androgen deprivation therapy. Fifteen patients had suffered from grade 2 or more (grade $2+$ ) late rectal bleeding. The numbers of predictors for a multivariable logistic regression NTCP model were determined by the least absolute shrinkage and selection operator (LASSO).

Results: The most important predictors for late rectal bleeding ranked by LASSO were platelet count, risk group and the relative volume of rectum receiving at least $65 \mathrm{~Gy}\left(\mathrm{~V}_{65}\right)$. The NTCP model of grade 2+ rectal bleeding was as follows: $S=-17.49+$ Platelets $(1000 / \mu \mathrm{L}) *(-0.025)+$ Risk group * Corresponding coefficient (low-risk group $=0$; intermediate-risk group $=19.07$; high-risk group $=20.41)+V_{65} * 0.045$.

Conclusions: A LASSO-based multivariable NTCP model comprising three important predictors (platelet count, risk group and $\mathrm{V}_{65}$ ) was established to predict the incidence of grade 2+ late rectal bleeding after IMRT.
\end{abstract}

Key words: rectal bleeding; radiation therapy; normal tissue complication probability; prostate cancer

\section{Introduction}

Radiation therapy is one of important treatment methods for patients with localized prostate cancer. With modern intensity-modulated radiation therapy (IMRT), conformal coverage of target volumes with limited doses to surrounding normal organs could be achieved, which might contribute to less radiation toxicity in surrounding organs. However, some patients still suffered from chronic proctitis with moderate to severe rectal bleeding; consequently, not only dose-volume factors but also clinical factors, such as the platelet count or the history of taking anticoagulants, should be taken into consideration while evaluating the prognosticators of late rectal bleeding. 
How to select an appropriate number of predictors for the incidence of radiation toxicities from many dosimetric and clinical factors is a question that has been investigated previously. Recently, the use of least absolute shrinkage and selection operator (LASSO) method made it possible to construct a useful multivariable normal tissue complication probability (NTCP) model. The merits of LASSO consist of handling multicollinearity problem, smaller mean squared error, full-range variable selection and coefficient shrinkage [1, 2], and more over being easy to fulfill [3-7]. Reports from Lee et al. $[1,8,9]$ and $\mathrm{Xu}$ et al. [10, 11] also suggested using the LASSO-based method for the selection of multivariable logistic regression NTCP predictive factors.

Consequently, the purpose of this study was to develop a LASSO-based multivariable NTCP model to predict moderate to severe late rectal bleeding among patients with prostate cancer treated by IMRT.

\section{Materials and Methods}

\section{Patients}

The Chang Gung Medical Foundation Institutional Review Board approved this retrospective research and waived the requirement for informed consent (102-0480B). The eligibility criteria were as follows: male patients with age $\geq 18$ years, histologically confirmed prostate adenocarcinoma, neither nodal nor distant metastasis, curatively intent treated by IMRT, and Eastern Cooperative Oncology Group (ECOG) performance status 0-2. Androgen deprivation therapy prior to radiation is allowed. The exclusion criteria were as follows: previous prostatectomy history; previous pelvic radiation therapy history; previous systemic chemotherapy history; previous or concurrent other cancer history within five years. From 2008 to 2011, 75 patients with localized prostate cancer were eligible; however, 7 patients were excluded in this retrospective study due to lack of sound pre-treatment data or loss of follow-up within one year after IMRT. Finally, 68 patients were enrolled for the study and the characteristics were listed in Table 1 . The median follow-up time was 56 months (range 14-87 months).

\section{Radiation therapy}

Each patient was immobilized in a supine position by a thermoplastic cast from lower abdomen to thigh and underwent pelvic computed tomography (CT) simulation to acquire axial images with a slice thickness of 2.5 to $5.0 \mathrm{~mm}$ for IMRT planning. The clinical target volume (CTV) was delineated according to $\mathrm{D}^{\prime}$ Amico risk classification [12] of prostate cancer. For low-risk and intermediate-risk patients, the CTV comprised the prostate and seminal vesicles at the first treatment course, and the field was reduced to include prostate only at the second treatment course. For high-risk patients, the CTV comprised the prostate, seminal vesicles and pelvic lymph nodes at the first treatment course, and the field was reduced to include the prostate and seminal vesicles at the second treatment course. The CTV was expanded three-dimensionally with a 5-10 $\mathrm{mm}$ margin to build up the planning target volume (PTV). The normal organs (e.g., rectum, urinary bladder and small bowels) locating at the PTV level on the CT images were contoured for dose constraint. The prescribed dose was 1.8-2.0 Gy per fraction per day to achieve 44-50 Gy at the first treatment course, and the total dose was escalated to around 72 Gy at the second treatment course.

Each IMRT plan adopted 6-8 coplanar portals to deliver 6-10 MV photons. The planning system was Pinnacle3 (Philips Medical Systems, Madison, WI). The prescribed dose must cover $95 \%$ of the PTV, and the small hot spot area over $110 \%$ of the prescribed dose should be limited to within the PTV. The surrounding organ dose-volume constraints were usually as follows: the relative volume of rectum receiving dose over 70 Gy should not be more than $25 \%$; the max dose in the small bowel should be limited to $55 \mathrm{~Gy}$. As for the urinary bladder, at least one half of the volume kept the dose no more than 65 Gy.

Forty-one patients also underwent image-guided radiation therapy (IGRT) at their expense. For IGRT, the slice thickness of planning CT images was reduced from $2.5 \mathrm{~mm}$ to $1.25 \mathrm{~mm}$, and the Novalis system (Varian Medical Systems, Palo Alto, CA, and BrainLAB, Heimstetten, Germany), a dedicated linear accelerator designed for stereotactic radiosurgery was applied. We have described the details of this image-guided stereotactic radiosurgery system previously [13]. Every day before treatment, the bony landmark of pubic bone was checked for revision of the inter-fractional movements during the treatment course. In addition, some of the patients undergoing IGRT were implanted with fiducial markers for tracking in the prostate one week before CT simulation.

\section{Androgen deprivation therapy (ADT)}

The usage of ADT together with IMRT was also according to the D'Amico risk classification of prostate cancer. For low-risk patients, they underwent local radiation therapy alone without ADT. For intermediate-risk patients, short-term subcutaneous injection of leuprorelin acetate $3.75 \mathrm{mg}$ per month had 
been prescribed for 4-6 months combined with IMRT. For high-risk patients, long-term subcutaneous injection of leuprorelin acetate $3.75 \mathrm{mg}$ every one month or 11.25 mg every 3 months had been given for 2 years. Oral anti-androgen medication, such as bicalutamide $50 \mathrm{mg}$, had also been prescribed per day during the first week of ADT.

\section{Follow-up}

After treatment, patients received periodic follow-up until death or lost to follow-up. They came back to our clinics every 1-3 months after IMRT in the first 2 years, every 3-6 months in the third to fifth year and annually after five years. History taking assessed urologic discomfort, diarrhoea, rectal bleeding and bone pain. Prostate-specific antigen (PSA) level was checked at each visit and image studies were performed when PSA level elevated or clinic indications dictated. Radiation toxicities such as urinary urgency, frequency, diarrhoea and rectal bleeding were recorded.

\section{Late rectal bleeding}

According to the medical records in the follow-up clinics, late rectal bleeding was identified as the occurrence of blood passing from anus or bloody stool three months after the completion of radiation therapy or later. The severity of late rectal bleeding was retrospectively graded according to the Common Terminology Criteria for Adverse Events (CTCAE) version 4.0, which defined it as follows: grade 1, mild without intervention; grade 2 , moderate with medical intervention or minor cauterisation; grade 3, severe with transfusion or aggressive intervention (radiologic, endoscopic, or operative intervention); grade 4, life-threatening with urgent intervention; grade 5, death. The patients with grade $2+$ rectal bleeding all underwent proctoscopy or colonoscopy to confirm the bleeding resulting from chronic radiation proctitis, which presented telangiectasia, ulceration, friability and contact bleeding or spontaneous bleeding in the rectal mucosa [14].

\section{Statistical analysis}

For the variables selection processing, 17 candidate risk factors were included initially for each patient. The clinical candidate factors were age, initial PSA, pre-treatment platelet count $(1000 / \mu \mathrm{L}), \mathrm{T}$ coding, risk group, ADT, IGRT, fiducial marker and anticoagulant. The dosimetric candidate factors were $\mathrm{V}_{30}-\mathrm{V}_{75}$ (relative rectal volumes receiving at least 30-75 Gy). The LASSO method was used to pick the optimal number of potential risk factors for the NTCP model. The following equation was used to erase the redundant parameters and choose the risk factors needed. (Tibshirani presented the LASSO algorithm in 1996) [15].

$$
\arg \min _{\beta}\|Y-X \beta\|_{\text {subject to }}^{2}\|\beta\|=\sum_{j=0}^{d}\left|\beta_{j}\right| \leq t
$$

where $t$ expresses the parameter that manages the grade of penalty, and $d$ is the number of variables chosen $[2,11]$.

The variables picked were used to build the multivariable logistic NTCP regression model. The predictive risk values were evaluated for each set of risk factors based on the NTCP regression coefficients by using the following formula $[1,11]$ :

$$
N T C P=\left(1+e^{-S}\right)^{-1}, \text { where } S=\beta_{0}+\sum_{i=1}^{n} \beta_{i} \cdot x_{i}
$$

where variable $\beta i$ is the relevant coefficient and $\mathrm{X} i$ is the different risk factors; $n$ expresses the number of risk factors.

Two datasets, a training set and a test set, were used to overcome the overfitting problem. A model was built based on a training set and fitted to the training set. It was also tested with a test set. We used nested 10-fold cross-validation to obtain the best prognostic factor subsets [11]. The system performance for the multivariable logistic NTCP regression model was calculated by using the area under the receiver operating characteristic curve (AUC), Omnibus, Nagelkerke's $\mathrm{R}^{2}$ and Hosmer-Lemeshow test $[2,8]$. For a review, see refs. $[1,8,9,16,17]$. SPSS version 19.0 (SPSS, Chicago, IL, USA) was used for the statistical analyses.

\section{Results}

Fifteen patients suffered from grade 2 or more (grade 2+) late rectal bleeding (grade 2: 6; grade 3: 6; grade 4: 2; grade: 1). The median time to develop grade $2+$ rectal bleeding after IMRT was 17.7 months. Six patients (4 patients with grade 2 bleeding and 2 patients with grade 3 bleeding) were successful to stop bleeding before last follow-up. The clinical candidate predictors for late rectal bleeding and the dosimetric parameters of rectal dose-volume relationship, $\mathrm{V}_{30}-\mathrm{V}_{75}$, were listed in Table 1 .

Seventeen clinical and dosimetric factors probably correlated with grade $2+$ rectal bleeding were ranked by LASSO and the result was listed in Table 2. The system performance evaluation of NTCP models in different numbers of optimal predictive factors calculated by AUC and Hosmer-Lemeshow test were shown in Table 3. The AUC was gradually increased from 0.78 to 0.85 and 0.87 while adding the second factor and the third factor; however, adding 
the fourth factor did not contribute to a greater AUC. Consequently, we selected three predictive factors, including platelets $(1000 / \mu \mathrm{L})$, risk group and $\mathrm{V}_{65}$, to develop the multivariable NTCP model of grade $2+$ rectal bleeding after IMRT, which as follows:

$S=-17.49+$ Platelets $(1000 / \mu \mathrm{L}) *(-0.025)+$ Risk group

* Corresponding coefficient $+\mathrm{V}_{65}$ * 0.045

The "Risk group * Corresponding coefficient" was shown as $\beta * X$ value (low-risk group $=0$; intermediate-risk group $=19.07$; high-risk group $=$ 20.41). The multivariable logistic regression coefficients and odds ratios for the NTCP model were listed in Table 4. We found that the pre-treatment platelet count was the most important predictor of grade $2+$ rectal bleeding.

Table 1. Clinical and dosimetric characteristics of the patient population

\begin{tabular}{|c|c|c|c|}
\hline Late rectal bleeding & All (\%) & Grade $0-1(\%)$ & Grade $2+(\%)$ \\
\hline Number & $68(100)$ & $53(78)$ & $15(22)$ \\
\hline \multicolumn{4}{|l|}{ Age (years) } \\
\hline Mean & 72 & 71 & 75 \\
\hline Range & $50-87$ & $50-86$ & $57-87$ \\
\hline \multicolumn{4}{|l|}{ Gleason score } \\
\hline $5-6$ & $26(38)$ & $19(36)$ & $7(47)$ \\
\hline 7 & $22(32)$ & $19(36)$ & $3(20)$ \\
\hline $8-10$ & $20(30)$ & $15(28)$ & $5(33)$ \\
\hline \multicolumn{4}{|l|}{ Initial PSA (ng/mL) } \\
\hline Mean & 37.24 & 37.10 & 37.70 \\
\hline Range & $1.60-235.50$ & $1.60-235.50$ & $8.10-98.50$ \\
\hline \multicolumn{4}{|l|}{ Platelets $(1000 / \mu \mathrm{L})$} \\
\hline Mean & 208.01 & 219.81 & 166.33 \\
\hline Range & $67-347$ & $133-347$ & $67-231$ \\
\hline \multicolumn{4}{|l|}{ T stage } \\
\hline $\mathrm{T} 1$ & $6(9)$ & $5(10)$ & $1(7)$ \\
\hline $\mathrm{T} 2$ & $51(75)$ & $42(79)$ & $9(60)$ \\
\hline $\mathrm{T} 3$ & $11(16)$ & $6(11)$ & $5(33)$ \\
\hline \multicolumn{4}{|l|}{ Risk group } \\
\hline Low & $9(13)$ & $9(17)$ & 0 \\
\hline Intermediate & $20(29)$ & $18(34)$ & $2(13)$ \\
\hline High & $39(58)$ & $26(49)$ & $13(87)$ \\
\hline \multicolumn{4}{|l|}{ ADT } \\
\hline No & $26(38)$ & $23(43)$ & $3(20)$ \\
\hline Yes & $42(62)$ & $30(57)$ & $12(80)$ \\
\hline \multicolumn{4}{|l|}{ IGRT } \\
\hline No & $27(35)$ & $21(40)$ & $6(40)$ \\
\hline Yes & $41(65)$ & $32(60)$ & $9(60)$ \\
\hline \multicolumn{4}{|l|}{ Fiducial marker } \\
\hline No & $57(84)$ & $46(87)$ & $11(73)$ \\
\hline Yes & $11(16)$ & $7(23)$ & $4(27)$ \\
\hline \multicolumn{4}{|l|}{ Anticoagulant } \\
\hline No & $40(59)$ & $33(62)$ & $7(47)$ \\
\hline Yes & $28(41)$ & $20(38)$ & $8(53)$ \\
\hline \multicolumn{4}{|l|}{ Rectum dose } \\
\hline $\mathrm{V}_{30}(\%)$ & 79 & 80 & 78 \\
\hline $\mathrm{V}_{40}(\%)$ & 67 & 67 & 70 \\
\hline $\mathrm{V}_{50}(\%)$ & 51 & 50 & 55 \\
\hline $\mathrm{V}_{55}(\%)$ & 42 & 41 & 47 \\
\hline $\mathrm{V}_{60}(\%)$ & 34 & 33 & 38 \\
\hline $\mathrm{V}_{65}(\%)$ & 26 & 25 & 31 \\
\hline $\mathrm{V}_{70}(\%)$ & 19 & 18 & 22 \\
\hline $\mathrm{V}_{75}(\%)$ & 9 & 8 & 12 \\
\hline
\end{tabular}

Abbreviations: $\mathrm{PSA}=$ prostate-specific antigen, $\mathrm{ADT}=$ androgen deprivation

therapy, IGRT $=$ image-guided radiation therapy, $\mathrm{V}_{30}-\mathrm{V}_{75}=$ mean relative volumes (\%) receiving at least $30-75 \mathrm{~Gy}$.
Table 2. Probable predictors ranked by LASSO

\begin{tabular}{|c|c|c|c|c|c|}
\hline Ranking & Predictor & Ranking & Predictor & Ranking & Predictor \\
\hline 1 & Platelets & 7 & Anticoagulant & 13 & ADT \\
\hline 2 & Risk group & 8 & Initial PSA & 14 & $\mathrm{~V}_{40}$ \\
\hline 3 & $\mathrm{~V}_{65}$ & 9 & $\mathrm{~V}_{55}$ & 15 & $\mathrm{~V}_{70}$ \\
\hline 4 & T stage & 10 & $\mathrm{~V}_{30}$ & 16 & $\mathrm{~V}_{60}$ \\
\hline 5 & Age & 11 & $\mathrm{~V}_{75}$ & 17 & $\mathrm{~V}_{50}$ \\
\hline 6 & Fiducial Marker & 12 & IGRT & & \\
\hline
\end{tabular}

Table 3. System performance evaluation according to number of predictors

\begin{tabular}{|c|c|c|c|c|}
\hline $\begin{array}{l}\text { Number of } \\
\text { predictors }\end{array}$ & AUC (95\% CI) & $\mathrm{R}^{2}$ Nagelkerke & Omnibus & $\begin{array}{l}\text { Hosmer - } \\
\text { Lemeshow }\end{array}$ \\
\hline 1 & $0.78(0.65-0.90)$ & 0.29 & 0.001 & 0.43 \\
\hline 2 & $0.85(0.75-0.95)$ & 0.41 & 0.001 & 0.42 \\
\hline $3^{*}$ & $0.87(0.79-0.96)$ & 0.45 & 0.001 & 0.62 \\
\hline 4 & $0.87(0.79-0.96)$ & 0.46 & 0.001 & 0.83 \\
\hline
\end{tabular}

Table 4. Multivariable logistic regression coefficients and odds ratios

\begin{tabular}{lllll}
\hline Predictive factors $(\mathrm{n}=3)$ & $\beta$ & $p$-value & $\mathrm{OR}$ & $95 \% \mathrm{CI}$ \\
\hline Platelet count $(1000 / \mu \mathrm{L})$ & -0.025 & 0.01 & 0.975 & $0.96-0.99$ \\
Risk Group & & & & \\
Risk Group (Intermediate versus Low) & 19.07 & 0.99 & & \\
Risk Group (High versus Low) & 20.41 & 0.99 & & \\
$\mathrm{~V}_{65}$ & 0.045 & 0.16 & 1.05 & $0.98-1.12$ \\
Constant & -17.49 & 0.99 & & \\
\hline Abbreviations: $\mathrm{NTCP}=$ normal tissue complication probability, $\mathrm{V}_{65}=$ relative rectal \\
volume (\%) receiving at least 65 Gy, OR = odds ratio, CI = confidence interval
\end{tabular}

\section{Discussion}

We established a multivariable NTCP model for the incidence of late moderate-to-severe rectal bleeding of patients with prostate cancer definitively treated by IMRT. Unlike many other studies [18-22], which almost built up only univariate NTCP models to fit their rectal bleeding data, this study developed a multivariable model, comprising two clinical predictors and one dosimetric predictor, with good system performance (AUC value: 0.87). With the LASSO method, we could shrink the model coefficients and reduce variances, resulting in superior overall prediction performance $[1,2]$.

Recently, Schaake et al. [23] also reported the predictors for late complications after IMRT in prostate cancer patients. They focused on the role of different anatomical subregions within and around the anorectum in three multivariable NTCP models for rectal bleeding, stool frequency and fecal incontinence. The predictors for late rectal bleeding in their study comprised both the use of anticoagulants and the percentage of relative anorectum volume receiving at least $70 \mathrm{~Gy}\left(\mathrm{~V}_{70}\right)$. The different outcome of dosimetric predictor was not so surprising as their 
study investigated the anorectum instead of the entire rectum. Moreover, $V_{65}$ and $V_{70}$ were most likely highly correlated. The different outcome of clinical predictor might be due to the different candidates included for analysis. Their candidates included history of diabetes, smoking, cardiovascular disease and abdominal surgery, but did not include risk group classification and pre-treatment platelet count. Consequently, the use of anticoagulants might play the leading role in the absence of the data regarding platelets.

The rectal mucosa in chronic radiation proctitis becomes fragile and ulcerative, which makes it more susceptible to contact bleeding or spontaneous bleeding. In the initial process of hemostasis, platelets adhere to the damaged vessel site and aggregate with extracellular ligands and soluble proteins to form platelet thrombi to stop bleeding [24]. Our study found that low platelet count was the most significant predictor. Since platelets were a key component in the blood and responsible for primary hemostasis, the low platelet count contributed to moderate-to-severe rectal bleeding in patients with fragile rectal mucosa due to radiation proctitis.

Our study also found that risk group was another clinical predictor. The patients were classified into different risk group according to D'Amico risk classification [12] criteria and underwent different IMRT field coverage and dose. Anatomically, the seminal vesicles were in the posterosuperior portion of the prostate and in front of the rectum. The irradiation boost to the seminal vesicles in high-risk group patients would increase the volume of the anterior rectal wall to receive a high radiation dose, which might contribute to the occurrence of radiation proctitis and late rectal bleeding.

Our study found that $V_{65}$ was an important dosimetric predictor. Several previous studies also proposed similar dose-volume constraints. One review article from Fiorino et al. [25] revealed that the dosimetric predictors were intermediate-to-high doses distributed from $V_{40}$ to $V_{78}$. In a Dutch phase III trial [26], anorectal $V_{65}$ was found to be the most significant parameter for late rectal bleeding. Likewise, Michalski et al. [27] proposed that the patients in the Radiation Therapy Oncology Group (RTOG) 9406 trial with $\mathrm{V}_{65}>50 \%$ had more common grade 2+ late bowel effects. A report from Fonteyne et al. [28] suggested the constraint of $\mathrm{V}_{65}<48 \%$ to prevent grade $2+$ lower intestinal toxicity. Someya et al. [29] also proposed that $\mathrm{V}_{65} \geq 17 \%$ significantly increased the risk of grade 2 or 3 rectal bleeding.

The incidence of grade $2+$ bleeding rate in our cohort was $22 \%$ in the median follow-up of nearly five years (56 months). The incidence rate was relatively high compared to other studies. Schaake et al. [23] from the Netherlands reported that $4.7 \%$ of patients experienced grade $2+$ rectal bleeding in the minimal follow-up of 3 years. Zelefsky et al. [30] reported that the 10-year grade 2+ bleeding rate was $5.0 \%$ at Memorial Sloan-Kettering Cancer Center (MSKCC). Takemoto et al. [31] from Japan reported that $8.2 \%$ of patients developed grade $2+$ rectal bleeding in the median follow-up of 35 months. Nevertheless, Someya et al. [29] also reported a 5-year incidence rate of grade 2 or 3 rectal bleeding of $21.6 \%$, which was compatible with our result. The different results between studies might be due to different patient populations, clinical conditions, immobilization and simulation techniques, and treatment planning details. For example, the patients underwent immobilization in the prone position at MSKCC [30]; however, our study cohort and the patients at other hospitals [23, 31] were immobilized in the supine position.

There were several limitations in this study. First, the number of patient population in this study was relatively small. Some candidate predictors might become insignificant due to low statistical power. Second, the subjective grading of rectal bleeding was according to the medical records retrospectively, which might lead to some degree of bias. Third, this multivariable NTCP model should need another cohort to validate the result in the future.

\section{Conclusions}

This study developed a LASSO-based multivariable NTCP model comprising three important predictors (platelet count, risk group and $\mathrm{V}_{65}$ ) to predict the incidence of grade 2+ late rectal bleeding in prostate cancer patients after IMRT. The low pre-treatment platelet count was the most significant predictor. In the future, we should prospectively validate the result of this model by an independent cohort.

\section{Acknowledgments}

This work was supported by the Ministry of Science and Technology, Taiwan (MOST-106-2221-E151-010), and the Chang Gung Memorial Hospital, Chang Gung Medical Foundation (CMRPG8C1141-3).

\section{Competing Interests}

The authors have declared that no competing interest exists.

\section{References}

1. Lee TF, Liou MH, Huang YJ, Chao PJ, Ting HM, Lee HY, et al. LASSO NTCP predictors for the incidence of xerostomia in patients with head and neck squamous cell carcinoma and nasopharyngeal carcinoma. Sci Rep. 2014; 4: 6217. 
2. Xu CJ, van der Schaaf A, Schilstra C, Langendijk JA, van't Veld AA. Impact of statistical learning methods on the predictive power of multivariate normal tissue complication probability models. Int J Radiat Oncol Biol Phys. 2012; 82: e677-84.

3. Colombani C, Legarra A, Fritz S, Guillaume F, Croiseau P, Ducrocq V, et al. Application of Bayesian least absolute shrinkage and selection operator (LASSO) and BayesCpi methods for genomic selection in French Holstein and Montbeliarde breeds. J Dairy Sci. 2013; 96: 575-91.

4. $\mathrm{Xu}$ J, Yin J. Kernel least absolute shrinkage and selection operator regression classifier for pattern classification. IET Computer Vision. 2013; 7: 48-55.

5. Ramsey SD, Andersen MR, Etzioni R, Moinpour C, Peacock S, Potosky A, et al. Quality of life in survivors of colorectal carcinoma. Cancer. 2000; 88: 1294-303.

6. Stock JH, Watson MW. Generalized Shrinkage Methods for Forecasting Using Many Predictors. Journal of Business \& Economic Statistics. 2012; 30: 481-93.

7. Teppola P, Taavitsainen VM. Parsimonious and robust multivariate calibration with rational function Least Absolute Shrinkage and Selection Operator and rational function Elastic Net. Anal Chim Acta. 2013; 768: 57-68.

8. Lee TF, Chao PJ, Ting HM, Chang L, Huang YJ, Wu JM, et al. Using multivariate regression model with least absolute shrinkage and selection operator (LASSO) to predict the incidence of Xerostomia after intensity-modulated radiotherapy for head and neck cancer. PLoS One. 2014; 9: $\mathrm{e} 89700$.

9. Lee TF, Huang EY. The different dose-volume effects of normal tissue complication probability using LASSO for acute small-bowel toxicity during radiotherapy in gynecological patients with or without prior abdominal surgery. Biomed Res Int. 2014; 2014: 143020.

10. van der Schaaf A, Xu CJ, van Luijk P, Van't Veld AA, Langendijk JA, Schilstra C. Multivariate modeling of complications with data driven variable selection: guarding against overfitting and effects of data set size. Radiother Oncol. 2012; 105: 115-21.

11. Xu CJ, van der Schaaf A, Van't Veld AA, Langendijk JA, Schilstra C Statistical validation of normal tissue complication probability models. Int J Radiat Oncol Biol Phys. 2012; 84: e123-9.

12. D'Amico AV, Whittington R, Malkowicz SB, Schultz D, Blank $K$, Broderick GA, et al. Biochemical outcome after radical prostatectomy, external beam radiation therapy, or interstitial radiation therapy for clinically localized prostate cancer. JAMA. 1998; 280: 969-74.

13. Lee TF, Chao PJ, Wang CY, Lan JH, Huang YJ, Hsu HC, et al. Dosimetric comparison of helical tomotherapy and dynamic conformal arc therapy in stereotactic radiosurgery for vestibular schwannomas. Med Dosim. 2011; 36: $62-70$

14. Vanneste BG, Van De Voorde L, de Ridder RJ, Van Limbergen EJ, Lambin P, van Lin EN. Chronic radiation proctitis: tricks to prevent and treat. Int J Colorectal Dis. 2015; 30: 1293-303.

15. Tibshirani R. Regression Shrinkage and Selection via the Lasso. Journal of the Royal Statistical Society Series B (Methodological). 1996; 58: 267-88.

16. Beetz I, Schilstra C, van der Schaaf A, van den Heuvel ER, Doornaert P, van Luijk P, et al. NTCP models for patient-rated xerostomia and sticky saliva after treatment with intensity modulated radiotherapy for head and neck cancer: the role of dosimetric and clinical factors. Radiother Oncol. 2012; 105: 101-6.

17. El Naqa I, Bradley J, Blanco AI, Lindsay PE, Vicic M, Hope A, et al. Multivariable modeling of radiotherapy outcomes, including dose-volume and clinical factors. Int J Radiat Oncol Biol Phys. 2006; 64: 1275-86.

18. Fiorino C, Cozzarini C, Vavassori V, Sanguineti G, Bianchi C, Cattaneo GM, et al. Relationships between DVHs and late rectal bleeding after radiotherapy for prostate cancer: analysis of a large group of patients pooled from three institutions. Radiother Oncol. 2002; 64: 1-12.

19. Cheung R, Tucker SL, Ye JS, Dong L, Liu H, Huang E, et al. Characterization of rectal normal tissue complication probability after high-dose external beam radiotherapy for prostate cancer. Int J Radiat Oncol Biol Phys. 2004; 58: 1513-9.

20. Peeters ST, Hoogeman MS, Heemsbergen WD, Hart AA, Koper PC, Lebesque JV. Rectal bleeding, fecal incontinence, and high stool frequency after conformal radiotherapy for prostate cancer: normal tissue complication probability modeling. Int J Radiat Oncol Biol Phys. 2006; 66: 11-9.

21. Sohn M, Yan D, Liang J, Meldolesi E, Vargas C, Alber M. Incidence of late rectal bleeding in high-dose conformal radiotherapy of prostate cancer using equivalent uniform dose-based and dose-volume-based normal tissue complication probability models. Int J Radiat Oncol Biol Phys. 2007; 67: 1066-73

22. Rancati T, Fiorino C, Fellin G, Vavassori V, Cagna E, Casanova Borca V, et al. Inclusion of clinical risk factors into NTCP modelling of late rectal toxicity after high dose radiotherapy for prostate cancer. Radiother Oncol. 2011; 100: $124-30$.

23. Schaake W, van der Schaaf A, van Dijk LV, Bongaerts AH, van den Bergh AC, Langendijk JA. Normal tissue complication probability (NTCP) models for late rectal bleeding, stool frequency and fecal incontinence after radiotherapy in prostate cancer patients. Radiother Oncol. 2016; 119: 381-7.

24. Versteeg HH, Heemskerk JW, Levi M, Reitsma PH. New fundamentals in hemostasis. Physiol Rev. 2013; 93: 327-58.

25. Fiorino C, Valdagni R, Rancati T, Sanguineti G. Dose-volume effects for normal tissues in external radiotherapy: pelvis. Radiother Oncol. 2009; 93: 153-67.

26. Peeters ST, Lebesque JV, Heemsbergen WD, van Putten WL, Slot A, Dielwart $\mathrm{MF}$, et al. Localized volume effects for late rectal and anal toxicity after radiotherapy for prostate cancer. Int J Radiat Oncol Biol Phys. 2006; 64: 1151-61

27. Michalski JM, Winter K, Purdy JA, Perez CA, Ryu JK, Parliament MB, et al. Toxicity after three-dimensional radiotherapy for prostate cancer with RTOG 9406 dose level IV. Int J Radiat Oncol Biol Phys. 2004; 58: 735-42.

28. Fonteyne V, De Neve W, Villeirs G, De Wagter C, De Meerleer G. Late radiotherapy-induced lower intestinal toxicity (RILIT) of intensity-modulated radiotherapy for prostate cancer: the need for adapting toxicity scales and the appearance of the sigmoid colon as co-responsible organ for lower intestinal toxicity. Radiother Oncol. 2007; 84: 156-63.

29. Someya M, Hori M, Tateoka K, Nakata K, Takagi M, Saito M, et al. Results and DVH analysis of late rectal bleeding in patients treated with 3D-CRT or IMRT for localized prostate cancer. J Radiat Res. 2015; 56: 122-7.

30. Zelefsky MJ, Levin EJ, Hunt M, Yamada Y, Shippy AM, Jackson A, et al. Incidence of late rectal and urinary toxicities after three-dimensional conformal radiotherapy and intensity-modulated radiotherapy for localized prostate cancer. Int J Radiat Oncol Biol Phys. 2008; 70: 1124-9.

31. Takemoto S, Shibamoto Y, Ayakawa S, Nagai A, Hayashi A, Ogino H, et al. Treatment and prognosis of patients with late rectal bleeding after intensity-modulated radiation therapy for prostate cancer. Radiat Oncol. 2012; 7: 87. 